(\$3.2 million) budget comes from the French Ministry of Education - an initiative started by President Georges Pompidou in 1965. Before that, IHES scraped along with relatively small subscriptions from a handful of business sponsors. But this year, only FF570,000 $(\$ 95,000)$ has been contributed by the private sector. Now, laments Marcel Berger, it is almost impossible to find money for basic research in industry. This has always depended on personal contacts established by the incumbent president, and can lapse when there is a change at the top.

Although genuinely international, IHES derives only 15 per cent of its income from European governments and is not eligible for European Commission support. Since 1971, the British Science and Engineering Research Council (SERC) and the West German Max Planck Gesellschaft have each given grants, this year of just over FF1 million $(\$ 166,000)$. The Swiss and Belgian governments have also supported IHES since 1971 , together providing about 5 per cent of its income, while the governments of Ireland, Denmark, the Netherlands, Finland, Portugal, Israel and Brazil also make modest contributions (between $\$ 2,000$ and $\$ 13,000$ ). The US National Science Foundation (NSF) has supported IHES since 1985 , this year giving $\$ 65,000$. This grant, a rare gesture for NSF, reflects the large number of US visitors to IHES, by far the majority.

But money is becoming an increasing worry for the institute, which started the year in deficit. "The private status of IHES gives us great flexibility and autonomy", says Marcel Berger. "The fellows' stipends, while they cannot match those available in the United States, aim to free them from money worries, while it is possible to organize a seminar or arrange a visit at the drop of a hat."

But, with the library - a former music room - cracking under the weight of its books and needing expensive underpinning, Berger is having to buy time by leaving vacancies unfilled. An important ally has been found in the European Science Foundation which, since 1976, has carried out a five-yearly review of the IHES scientific programme. The panel's review helps guarantee the institute's autonomy, while ensuring that its high standards and international connections are maintained. That, in turn, makes governments - and, in principle, industry - happier to continue their support.

Berger says it is "unthinkable" that IHES would ever be threatened with closure. But if any of its sponsors, such as SERC, should be prevented by national government policy from helping, would suffer and its sometimes fragile occupants could once again become an endangered species in Europe.

\title{
Grenoble as physics city
}

\section{Paris}

THE French city of Grenoble, near the Swiss and Italian borders, is, by a mixture of fate and design, becoming a 'Eurocity'. But unlike Brussels, Strasbourg or Luxembourg, Grenoble's cosmopolitanism stems not from politics but from a distinctive blend of unrivalled basic science facilities and new technology industry. The city's fourteenth-century university may already have had an international flavour, but twentieth-century physics has put Grenoble on the map of European science.

In 1946, the French national science research centre (CNRS) opened its first major laboratory outside Paris in the city, with its Service National des Champs Intenses. In 1956, the French atomic energy commission (CEA) chose Grenoble for its research establishment and in 1966 the Institut Laue-Langevin, which houses the world's highest-performance neutronbeam reactor, joined the family.

This year, construction has started on the latest, and most European, of Grenoble's big science instruments - the European Synchrotron Radiation Facility (ESRF). The idea that Europe should build its own major synchrotron facility was first put to the European Science Foundation in 1975. There followed a period of diplomacy, definition and costing until, in 1985, the two major promoters, France and West Germany, proposed to go ahead with the project.

At the end of 1985 , a memorandum of understanding was drawn up between France, Germany, Great Britain, Italy and Spain. In 1987 the protocol was signed, with Switzerland and a Nordic consortium joining the original five members. The formal convention and statutes alloowing money to be spent was signed in Paris on 16 December last year.

At present, the site is being excavated, but, says Karl Witte, assistant to ESRF's director (Ruprecht Haensel), "you can already see a few circles on the ground". The first seven of ESRF's beam lines are due to be opened in 1994, with a target date of 1998 for the full 30 planned. When completed, ESRF will be the world's most powerful radiation synchrotron, able to accelerate electrons or positrons up to 6 $\mathrm{GeV}$, with a beam width of about one tenth of a millimetre.

But the title may quickly be lost. A $7 \mathrm{GeV}$ synchrotron is to be built at the Argonne National Laboratory in Illinois and, last December, the Japanese government announced plans for a $8 \mathrm{GeV}$ synchrotron facility near Osaka.

The total cost of ESRF will be FF2,600 million (about $\$ 406$ million), with operating costs for 1994-98 estimated at FF1,000 million. France is contributing 34 per cent of the cost, and in addition has supplied the site and the local infrastructure. West Germany is the second largest partner, with a 24 per cent stake, followed by Italy (14.5 per cent) and Britain (12.5 per cent). The balance will shared between Spain, Switzerland and the Nordic countries (4 per cent each) with Belgium providing 3 per cent.

Last month, at the first ESRF users, meeting, 200 Europeans and a handful of colleagues from the United States, Japan and Israel, discussed priorities for the first seven beam lines and verified that the synchrotron's specification (called the 'red book') is still appropriate. Discussions will continue until the scientific advisory committee meeting at the end of June.

Although ESRF still has only a skeleton staff, there are already signs that it may be difficult to achieve a mix of nationalities in the resident staff (on five-year fixed con-

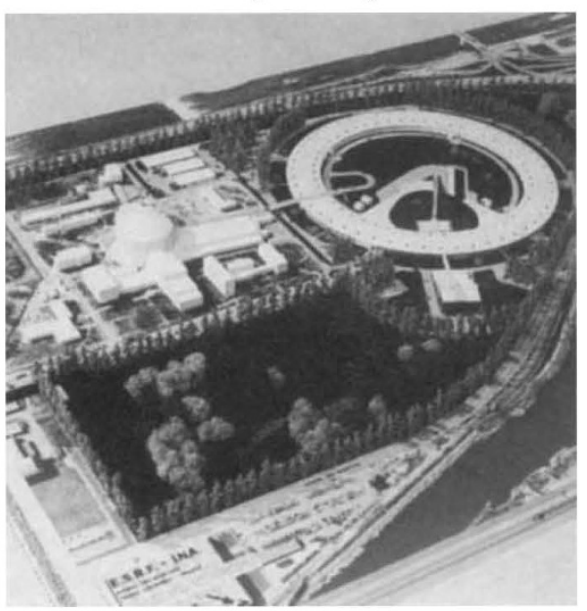

A model institute, but building is under way.

tracts) which reflects members' relative contributions. "We wanted to keep the French presence below 50 per cent", says Witte, but the proportion seems now to be about 52 per cent. The difficulty is that it is not easy to attract scientists and technicians from other member states because ESRF can offer only French salaries in line with those of the CEA. Constitutionally, ESRF is a French company and in that respect unlike, for example, CERN, where salaries are fixed on the international European scale.

More than 2,000 people are expected to use ESRF each year, with all costs paid for out of member states' contributions. Experiments will be selected on the basis of merit by a scientific panel on which each member state will be represented. If a "significant imbalance" emerges between a country's rate of use of the facility and its percentage contribution, the statutes provide for an eventual retrospective "readjustment" of payments. This principle may free referees assessing proposals for using the machine from the straitjacket of national time-sharing quotas.

P.C. 